Résumés des conférences et travaux

146 | 2015

2013-2014

\title{
Ecdotique des textes latins antiques
}

\section{Ecdotique des textes latins antiques}

Conférences de l'année 2013-2014

\section{Gauthier Liberman}

\section{OpenEdition \\ Journals}

Édition électronique

URL : https://journals.openedition.org/ashp/1703

DOI : 10.4000/ashp.1703

ISSN : 1969-6310

Éditeur

Publications de l'École Pratique des Hautes Études

Édition imprimée

Date de publication : 1 septembre 2015

Pagination : 81-94

ISSN : 0766-0677

Référence électronique

Gauthier Liberman, "Ecdotique des textes latins antiques ", Annuaire de l'École pratique des hautes études (EPHE), Section des sciences historiques et philologiques [En ligne], 146 | 2015, mis en ligne le 28 septembre 2015, consulté le 07 juillet 2021. URL : http://journals.openedition.org/ashp/1703 ; DOI : https://doi.org/10.4000/ashp.1703 


\title{
ECDOTIQUE \\ DES TEXTES LATINS ANTIQUES
}

\author{
Directeur d'études : M. Gauthier LiBERMAN
}

Programme de l'année 2013-2014 : I. Réflexion théorique. - II. Exercices critiques (passages de Virgile, Horace, Pline le Jeune).

\section{Réflexion théorique}

On a évoqué, dans la première séance, l'histoire de l'enseignement de la critique des textes et de l'ecdotique à l'EPHE et le nom et l'œuvre de ceux qui y ont enseigné spécifiquement la critique verbale des langues classiques et en particulier du latin. La première séance a aussi donné lieu à une présentation générale des modalités de la transmission des textes classiques qui justifient l'existence d'une critique verbale et conjecturale. On a aussi circonscrit rigoureusement ces notions de critique textuelle et de critique verbale, puis l'on est passé à la grande question d'une conférence d'ecdotique, question aussi simple dans son énoncé que complexe et controversée dans les réponses théoriques et pratiques et les débats qu'elle suscite : quel texte l'auteur d'une édition scientifique doit-il éditer? On a remarqué que la question se pose différemment selon le caractère oral ou écrit de la composition du texte, selon l'unicité ou la multiplicité de ses auteurs, selon l'histoire et la transmission du texte, selon les objectifs des éditeurs et les habitudes consacrées des différents champs disciplinaires, l'ecdotique n'étant pas transcendante à ces champs mais immanente à chacun d'eux. On a observé que les éditeurs de textes classiques montrent peu de propension à dire quel texte ils se proposent d'éditer : le texte de l'auteur ou celui qui s'en rapproche le plus possible, pour peu qu'il y ait un unique texte original ? le texte de l'archétype de la tradition s'il y a un archétype ? Il est apparu que la proposition « éditer le texte des manuscrits » n'a aucun sens dans la mesure où les manuscrits présentent des variantes : seul « éditer le texte d'un manuscrit » (de préférence un manuscrit sans variantes) a un sens, mais l'on constate aisément que les éditeurs de textes classiques n'éditent jamais le texte d'un manuscrit, ce qui serait une édition diplomatique ou quasi diplomatique mais non une édition critique ou scientifique. Posons que, dans la majorité des cas qui se présentent, le responsable d'une édition critique d'un classique latin doit s'efforcer d'éditer le texte tel que l'a écrit son auteur et tel qu'il figurait dans le manuscrit, autographe ou non, que nous admettons être, en dernière analyse, à l'origine absolue de la tradition textuelle. Imaginons qu'on propose ce but à plusieurs éditeurs ou à plusieurs équipes d'éditeurs : le résultat sera indubitablement fort différent, de telle manière qu'on reconnait une dimension subjective à l'appréciation du degré auquel une édition critique donnée réalise le but proposé à l'éditeur. Cette dimension, qui est une donnée irréductible du travail de l'éditeur et résulte des conditions générales et particulières de la transmission des textes, doit-elle amener à remettre en question le but que nous avons assigné à l'édition critique ? Éviterait-on la subjectivité en se proposant d'éditer 
le texte qui se rapproche le plus possible du texte transmis et qui ne sort pas du cercle des variantes autorisées? Mais le choix entre ces variantes a lui-même une dimension subjective qui n'est pas réduite par l'élévation d'un manuscrit ou d'une famille de manuscrit au statut de «meilleur manuscrit» ou " meilleure famille». La préférence donnée à une variante implique la reconnaissance du fait que la ou les variantes écartées sont fautives et que la tradition est par endroits fautive. Cela étant reconnu, les conditions de transmission des textes classiques rendent-elles vraisemblable qu'il n'y ait de fautes textuelles que celles que dénoncent des variantes fautives ? L'histoire de l'ecdotique montre que plus on a collationné de manuscrits, plus on a mis en évidence l'existence de leçons fautives : ce fait même ne parle guère en faveur d'une réponse positive à la question qui vient d'être posée. La mise en évidence du caractère fautif d'une variante ne diffère d'ailleurs pas de la mise en évidence du caractère fautif d'une leçon « unanimement transmise », pour reprendre une phraséologie aussi trompeuse que consacrée et qui, en comparant les manuscrits à des votants, implique souvent, chez ceux qui l'utilisent, l'idée qu'un vote unanime a toutes les chances d'être juste. $\mathrm{Si}$, dans les deux cas, la mise en évidence de la faute est la même, la faute sera-t-elle moins faute parce que la leçon suspecte est celle de tous les témoins ? La réponse est clairement négative, et pourtant il est indéniable qu'une variante jugée vraie n'a pas le même statut qu'une conjecture même jugée vraie, à moins qu'il s'agisse non d'une variante autorisée ou représentant un élément traditionnel mais d'une conjecture, quand bien même elle est attestée dans la tradition manuscrite. Faut-il donc cantonner toutes les conjectures dans l'apparat critique, parce qu'elles ne sont que des conjectures? Ce n'est pas une position intenable, si l'on estime que le lecteur doit considérer que dans une édition critique il y a interaction entre le texte et l'apparat critique. Alors, l'éditeur laisse au lecteur le soin de déterminer le texte qu'il juge vrai ou plausible et, ce faisant, lui abandonne une partie de son travail. Cette solution n'est guère qu'un pis-aller, mais un pis-aller qui peut ne pas être inutile, surtout dans le cas où le texte est très corrompu et où l'appareil infrapaginal constitue un commentaire critique. En effet, plus un texte est profondément altéré, plus la présence de très nombreuses corrections dans le texte masque la tradition, ce qui présente un inconvénient, non que cette tradition soit vraie mais parce que cette tradition suspecte échappera au lecteur, qui aura du mal à reconstituer mentalement le texte transmis. Housman a fait le contraire : dans le chef d'œuvre du genre, son commentaire critique du poème de Manilius, il admet ses conjectures dans le texte mais le texte qu'il édite dans une édition critique de peu postérieure et dépourvue de commentaire est beaucoup moins conjectural. Cela ne veut pas dire qu'il a changé d'avis : les deux travaux se complètent. Inversement, un commentaire critique appuyé sur un texte incluant peu ou pas de conjectures appelle une édition critique sans commentaire qui inclut les corrections auxquelles le critique accorde le degré de probabilité adéquat. C'est qu'éditer un texte que l'on considère fautif ou suspect ne saurait être un idéal, même si l'on met entre croix les leçons fautives, au risque de transformer un texte en cimetière. Cette solution, qui pourrait convenir à une édition critique sans traduction, présente naturellement de grands inconvénients dans les éditions accompagnées de traduction. Mais le pire serait de renoncer à indiquer les fautes dans le texte et même dans l'apparat et de traduire un autre texte que celui qui est édité. C'est le défaut dans lequel tombent bien des éditions 
dites critiques pourvues d'une traduction, et ce défaut est un risque inhérent à ce type d'éditions, bien loin que la présence d'une traduction garantisse une meilleure qualité du texte édité. C'est en effet que, parmi les intentions qui ont présidé à la création de notre collection nationale d'auteurs anciens, se trouve celle de prendre le contrepied de la pratique allemande consistant à faire des éditions critiques sans traduction. L'argument était que certains éditeurs de la collection Teubner eussent été bien en peine de justifier le texte édité par eux et ne l'auraient pas maintenu s'ils avaient eu à le traduire. Cet argument eût été plus recevable si, dans les faits, les éditeurs français, au lieu d'aligner la traduction sur le texte édité, n'avaient trop souvent traduit un texte différent du texte édité, réglant ainsi par des artifices de traduction des difficultés proprement textuelles. Ce n'est pas à dire qu'une traduction intelligente et inexacte ne soit pas utile à la critique verbale : au contraire, un traducteur doué peut mettre le critique textuel sur la voie d'une solution plausible en ayant aperçu le sens requis par le contexte. Inversement, la traduction très exacte d'un texte douteux peut aussi mettre la faute en évidence et contribuer à la solution.

Il y a donc, d'un côté, l'unicité de l'idéal, éditer le texte de l'auteur, retrouvé par le talent de l'éditeur aidé de celui de ses prédécesseurs (car il est bien connu que la capacité de reconnaître le mérite des bonnes conjectures de ses prédécesseurs est un des mérites de l'éditeur), et, de l'autre côté, la diversité des pratiques, qui tiennent compte de contingences : l'édition est-elle accompagnée ou non d'une traduction, la série exclut-elle les cruces desperationis ou les admet-elle, l'attitude ambiante estelle conservatrice ou portée à l'innovation conjecturale, les éditions antérieures d'un texte donné sont-elles marquées par le conservatisme ou son contraire? À tout cela s'ajoute le paramètre déjà évoqué de la subjectivité du jugement critique sous la forme de la diversité des appréciations portées sur une même correction : géniale et indispensable, certainement vraie selon l'un, futile et certainement fourvoyée d'après un autre, pour prendre les deux bouts du spectre. Comme on fait très souvent intervenir cette notion de subjectivité sans toujours réfléchir à ce qu'elle recouvre et implique, il vaut la peine d'y revenir. S'il est vrai que le jugement critique est subjectif et se ramène au jugement de goût de quo non disputatur, alors il faudrait éditer chaque version d'un texte transmis, puisque, nous l'avons vu, l'existence de variantes appelle un choix fondé sur l'exercice du jugement critique. Mais, dans ce jugement, la subjectivité est peut-être moins inhérente à la chose jugée qu'elle ne représente la part variable d'inadéquation d'un juge donné à la tâche qu'il doit remplir. La variabilité de cette adéquation selon les individus montre la difficulté de la tâche mais elle implique aussi, dans la chose jugée, un élément non objectif dont, pour faire bref, on admettra qu'il relève du goût. Dans les sciences dites dures, il est peut-être réservé à quelquesuns de faire des progrès décisifs et de fournir des démonstrations auxquelles se rallient les autres spécialistes; dans notre discipline, une conjecture vraie peut ne pas s'imposer parce que la reconnaissance de sa vérité objective requiert aussi ce je ne sais quoi dont un véritable critique ne saurait être dépourvu, un sens de l'adéquation qui ajuste au passage donné la connaissance des modes d'expression, de pensée et de sentir des auteurs anciens et la connaissance de la «pathologie textuelle». Cette dernière rassemble les modalités selon lesquelles se produisent les fautes qui déparent les texte et elle a naturellement fourni une ample matière aux conférences de 2013-2014. 
On a voulu prémunir les auditeurs contre une illusion qui, au moins dans les premières décennies du $\mathrm{Xx}^{\mathrm{e}}$ siècle, a contribué à ruiner les progrès de la science française en matière de critique verbale et d'ecdotique : les érudits ont cru pouvoir fonder sur le Manuel de Havet une «méthode » critique consistant en l'application de recettes mécaniques pour donner une solution aux problèmes textuels. Cette illusion fait encore aujourd'hui des dégâts, et pas seulement en France. Elle sévissait ailleurs avant que le Manuel de Havet n'exerce une influence, et elle est le fruit de l'intention d'assujettir la critique verbale et conjecturale à une méthode «scientifique » d'où l'incertitude subjective serait bannie et qui pourrait remplacer le jugement nourri par les connaissances adéquates. Au nom d'une rationalité positiviste, on traite donc les problèmes de texte d'une façon en réalité irrationelle : au lieu de rechercher le ou les mots que le contexte appelle et qui est / sont conforme(s) à la manière de l'auteur et aux us de la langue, on joue avec les lettres et les jambages de la leçon transmise ou des leçons transmises pour obtenir un résultat conforme à une « vraisemblance paléographique » a priori ou aux prétendues " règles de la méthode critique ». C'est poser les critères de la solution ou expliquer la faute avant d'avoir la solution! Une telle «méthode» ne saurait se réclamer ni de la méthode, ni de la science, ni de Louis Havet, même s'il est peu douteux que, en posant en principe l'explicabilité absolue de toutes les fautes textuelles et en rédigeant un manuel qui illustre tous les types de fautes, Havet n'ouvre malgré lui la voie aux errements de ceux qui croient être ses disciples. En réalité, les processus de faute peuvent être si complexes qu'il est possible de proposer une solution convaincante à un problème très difficile sans pouvoir arrêter un scenario simple et unique du processus qui a conduit au texte / aux textes transmis. La critique conjecturale est-elle pour autant le royaume de l'arbitraire? Non, bien qu'elle ne puisse consister dans l'application mécanique de recettes. Doit-on se passer le plus possible de la critique conjecturale, dans la mesure où elle ne peut donner lieu à l'application des règles d'une méthode a priori ? Autant dire que l'histoire qui ne se limite pas à l'établissement des faits est arbitraire et que pour cette raison il faut renoncer à une histoire plus ambitieuse. Et n'y a-t-il pas le plus gênant des paradoxes à inventer, pour réduire la part d'arbitraire le plus possible, une « méthode critique » elle-même irrationnelle? Non, il faut accepter les risques de la critique conjecturale. Rien de grand dans les sciences humaines ne s'est effectué sans risques et rien ne peut plus nuire à leur progrès que la réduction des processus d'invention à l'application mécanique de recettes. Le temps (parfois, surtout dans le domaine grec, sous la forme de l'apparition de nouveaux documents confirmant des corrections anciennes), le progrès $\mathrm{du}$ savoir et la maturation du jugement feront le tri entre le bon et le mauvais, à condition d'accepter les risques de l'exercice critique, sans lequel la faculté de juger s'atrophie au point de devoir renoncer à la tâche critique et de limiter le travail de l'éditeur à la recensio lachmanienne en sacrifiant la seconde partie, rassemblée par Lachmann sous le vocable d'emendatio. C'est ainsi qu'on finit par éditer, dans un passage discuté plus bas, habet hoc primum magna fortuna quod nihil tectum, nihil occultum esse patitur, et par traduire non primum mais la leçon dont la redécouverte conjecturale et néanmoins certaine aurait dû être l'apanage des seuls humanistes. 


\section{Exercices critiques}

En faisant la part belle aux exercices critiques on a non seulement, avec plaisir, cédé à ce qui fut jadis une belle tradition de l'EPHE $^{1}$, mais on a aussi saisi une occasion unique de former ou d'exercer l'esprit critique des auditeurs plus ou moins jeunes. On s'est ainsi penché sur de nombreux passages du livre II du poème de Lucain ${ }^{2}$ : la conférence comptait, parmi ses auditeurs assidus, M. Florian Barrière, à présent maître de conférences à l'université de Grenoble, qui avait soutenu brillamment une thèse comportant l'édition critique et commentée de ce même livre du Bellum Ciuile. On a aussi discuté des passages des poèmes de Paulin de Nole, dont un autre auditeur assidu, M. Franz Dolveck, à présent membre de l'École française de Rome, avait terminé l'édition critique. En confrontant leurs intelligences pénétrantes et subtiles à la fois l'une à l'autre et aux difficultés qui leur étaient soumises, les deux jeunes gens ont, à n'en pas douter, aiguisé leur esprit critique et forcé l'admiration de celui qui dirigeait le débat et des autres auditeurs, dont $\mathrm{M}^{\text {lle }}$ Carole Hofstetter, doctorante helléniste de l'EPHE. Au cours de la discussion sont nées des observations et idées nouvelles, parfois inattendues, qui confirment l'importance, même dans un domaine où la réflexion et l'invention personnelles comptent beaucoup, de la confrontation des intelligences et accusent le regret qu'on peut avoir de la difficulté qu'il y a à réunir ceux qui, par la force des choses, sont appelés un jour ou l'autre, même si ce n'est pas leur vocation initiale, à faire de la critique des textes. Qu'on le veuille ou non, les textes sont la base de tout dans les disciplines historiques et philologiques de l'Antiquité, les documents non textuels sont muets sans les textes, et n'importe quel historien et philologue, n'importe quel latiniste, devraient avoir une formation en critique textuelle : c'est moins que jamais le cas, en France et même dans les pays qui jadis et même naguère prisaient la critique verbale au point exagéré d'en faire dépendre ou d'y sacrifier les autres disciplines. Les jeunes générations de chercheurs se lancent dans des travaux philologiques, voire des commentaires de textes et des éditions, en n'ayant aucune idée précise de la façon dont les textes ont pu souffrir au cours de la transmission et des moyens dont on dispose pour remédier à cette pathologie. Il y a là un phénomène désastreux, qui est aussi fâcheux pour les chercheurs qui ne sont pas philologues et bâtissent des raisonnements sur des textes dont l'épaisseur philologique, la fragilité et la labilité leur échappent plus ou moins complètement.

On a accordé une importance particulière à des types de fautes connus mais négligés. Ainsi, au tout premier rang, la faute "à plusieurs degrés " consistant en la réparation erronée du dégat causé par la perte accidentelle d'un mot ou d'une séquence. C'est un type de fautes qui se rencontre particulièrement en poésie, où la perte d'un mot affecte le vers. Il est particulièrement propre à mettre en évidence le caractère trompeur de la trop facile comparaison souvent faite entre l'ecdotique innovante qui

1. Nous avons étudié les passages théoriques du recueil d'Édouard Tournier, Exercices critiques de la conférence de philologie grecque, $1^{\text {er }}$ août 1872 - $1^{\text {er }}$ août 1875, Paris, 1875, et regretté que les érudits ignorent les suggestions de Tournier, auxquelles nous aurons l'occasion de rendre justice dans des travaux portant sur Thucydide.

2. On a discuté et parfois critiqué certaines suggestions, dues à l'auteur de ces lignes, publiées par feu Georg Luck dans son édition-traduction de Lucain (Stuttgart, Reclam, 2009). 
ne répugne pas à la critique conjecturale et certaines restaurations archéologiques du $\mathrm{XIX}^{\mathrm{e}}$ siècle. Selon cette comparaison, pour retrouver le texte authentique, il suffirait d'effacer les restaurations abusives et arbitraires des philologues modernes, comme on élimine les restaurations de certaines églises médiévales ou de statues antiques. Mais la vérité est que l'original prétendu qui se trouve sous ces restaurations est lui-même une restauration nullement différente des restaurations modernes, à ceci près que les restaurations modernes sont indiquées dans un apparat critique et que ces restaurations anciennes constituent le texte que l'on prétend original sans être accompagnées d'un appareil critique qui les signale à l'attention du lecteur. Parce que, du moins à la connaissance de leur auteur, ils donnent lieu à des solutions le plus souvent nouvelles, on met ici en exergue les exemples suivants, dont le premier figure dans un texte consacré entre tous :

I Virgile, Bucoliques, 4.

Molli paulatim flauescet campus arista 28

incultisque rubens pendebit sentibus uиa

et durae quercus sudabunt roscida mella.

Comme la poussée de l'épi dans la plaine n'est pas un miracle et que la mollesse de l'épi ne peut pas constituer le miracle de la seconde étape, on est obligé de forcer le texte en entendant par campus « champ / plaine inculte ». Le latin poétique est souple, mais non au point qu'on puisse tirer incultus campus à partir de incultis sentibus au vers suivant. Or, s'il manque au v. 28 une épithète de campus, il s'y trouve un mot dont l'absence ne serait pas dommageable : paulatim. Je suggère que paulatim est un bouche-trou destiné à réparer la disparition d'une épithète de campus perdue entre molli et flauescet, et que cette épithète est siluestris. La cohérence du passage est alors parfaite et sa construction achevée : trois vers qui sont autant de propositions, trois verbes centraux $(\mathrm{C})$, trois substantifs sujets $(\mathrm{A})$ accompagnés chacun d'une épithète (B), trois compléments (D) accompagnés chacun d'une épithète (E), avec une légère variatio au second vers et une autre plus conséquente au troisième vers : $\mathrm{EB} C \mathrm{AD} \mid$ EB C DA | BA C ED. L'opposition entre siluestris et cultus se trouve, à propos de ager, chez Columelle, De re rust., 1 pr. 25, atque in his ipsis haberet cognitum, quid recusaret collis, quid campestris positio, quid cultus, quid siluestris ager, quid umidus et graminosus, quid siccus et spurcus. Siluestris est épithète de Musa en Buc. 1,2; de arbor en 3,70 et de labrusca en 5,7. Virgile, Géorg., 2,183, parle de strati bacis siluestribus agri.

II Horace, Satires, I.

Denique sit finis quaerendi, quoque habeas plus, pauperiem metuas minus, et finire laborem incipias parto quod auebas, ne facias quod Vmmidius quidam. Non longa est fabula : diues

ut metiretur nummos, ita sordidus ut se non umquam seruo melius uestiret, adusque supremum tempus ne se penuria uictus opprimeret metuebat. 
Tel est le texte de l'édition de Shackleton Bailey (Teubner Stuttgart, 1995³). Dans son commentaire (Cambridge Greek and Latin Classics, 2012) ${ }^{1}, \mathrm{M}^{\mathrm{me}} \mathrm{E}$. Gowers adopte le même texte, si ce n'est qu'elle écarte au v. 92 la conjecture de Muret quoque au profit de la leçon transmise et vulgaire, cumque (c'est-à-dire quomque), au motif que « craignons d'autant moins la pauvreté qu'on est plus riche » irait « against the speaker's philosophy ». Gowers explique que quidam (v. 95) est « a story-telling word » et, à propos de metiretur, note que « weighing or measuring money is proverbial for not being able to count it: cf. Xen. Hell. 3.2.27, Petr. Sat. 37 Fortunata appellatur, quae nummos modio metitur ». Quelles études faut-il faire pour s'étonner que mesurer son argent puisse être une expression proverbiale exprimant l'idée qu'on en a tant qu'on ne peut pas le compter? Le rapprochement des passages de Xénophon et Pétrone confirme l'intuition que quidam est un bouche-trou substitué au mot manquant, $M O D I O$, absorbé par umMIDIVS. Le texte et la ponctuation du passage doivent être les suivants :

ne facias quod

Vmmidius : modio (non longa est fabula) diues

ut metiretur nummos, ita sordidus ut se, etc.

Si elle est moins exposée à ce type de fautes, la prose ne les ignore pas. On a discuté les exemples suivants :

III Pline le Jeune, Panégyrique de Trajan, 1,5.

Non enim occulta potestate fatorum, sed ab Ioue ipso coram ac palam repertus electus est: quippe inter aras et altaria, eodemque loci quem deus ille tam manifestus ac praesens quam caelum ac sidera insedit.

Tel est le texte transmis, édité par R. A. B. Mynors (OCT, 1964) avec la ponctuation que l'on voit. Repertus electus est forme une suite problématique. Le texte de M. Durry (CUF, 1947), qui admet la correction de C. G. Schwartz, repertus est : electus quippe, représente un progrès mais un progrès modeste, car repertus, qui irait très bien avec occulta potestate fatorum, ne va pas bien avec coram ac palam, avec quoi electus cadrerait très bien. Comparer 94,4 (Pline s'adresse à Jupiter Capitolin), $T u$, uoce imperatoris quid sentires locutus, filium illi, nobis parentem, tibi pontificem maximum elegisti, et voir J. Béranger, Principatus. Études de notions et d'histoire politiques dans l'Antiquité gréco-romaine, Genève, 1973, p. 433 n. 22. On suggère donc :

Non enim occulta potestate fatorum repertus, sed ab Ioue ipso coram ac palam electus est : quippe <electus $>$ inter aras et altaria, etc.

Pour la répétition de electus, comparer Lettres, 8,6,4, offeruntur (quippe offeruntur a seruis). La clausule ac pal(am) electus est constitue un double crétique (cf. par exemple se|curior factus est 6,5). Plusieurs scenarii peuvent expliquer la faute, qui comporte le déplacement d'un mot et la réduction à une seule des deux occurrences d'un autre.

1. En rédigeant l'article Problèmes d'exégèse et de critique verbale horatiennes paru dans les Mélanges Lucienne Deschamps, O. Devillers et G. Flamerie de Lachapelle (éd.), Bordeaux, 2013, p. 193-211, je ne connaissais pas le commentaire de $\mathrm{M}^{\mathrm{me}}$ Gowers. Je ne peux m'empêcher de m'étonner qu'une latiniste de Cambridge ne mentionne pas Housman à propos de Satires, 1,8,36, passage que j'étudie dans cet article, p. 210-211. 
IV Panégyrique, 50,4 et 50,3.

Muta quidem illa et anima carentia sentire tamen et laetari uidentur quod niteant, quod frequententur, quod aliquando coeperint esse domini scientis.

Trajan restitue à des particuliers, pour qu'ils les habitent, les domaines quae priores principes occupabant (lire occuparant), non ut ipsi fruerentur, sed ne quis alius. Durry (édition commentée, Paris, 1938) explique scientis « qui connaît son domaine (ep. 1, $24,4)$ ou plutôt qui en connaît la valeur ». Mais scio seul semble par trop vague et chacune des deux interprétations laisserait attendre un complément exprimé. La première explication convient bien au contexte mais, si l'on ne s'abuse, correspond plutôt à noscentis, qui, lui, permet de sous-entendre naturellement le COD se, " un maître qui se familarise avec eux, apprend à les connaître » : le maître décrit par Pline dans le passage des Lettres évoqué par Durry est de ceux qui omnesque uiteculas suas nosse et numerare arbusculas possint. C. G. Schwartz citait aussi le célèbre passage où Trimalcion $(48,2)$ évoque l'un des domaines quod ego adhuc non noui. La seconde interprétation, préférée par Durry lui-même, pourrait correspondre à $<$ bona sua $>$ scientis (cf. l'oncle de Pline, 8,91, sciuntque non sua modo commoda uerum et hostium aduersa). Grâce au nouveau prince, nec iam clarissimorum uirorum receptacula habitatore seruo teruntur aut foeda uastitate procumbunt. Datur intueri pulcherrimas aedes deterso situ auctas ac uigentes (50,3). Lire uetustate (cf. par ex. Lettres, 6,30,2).

\section{Panégyrique, 62,3.}

An parum saepe experti[s] sumus hanc esse rerum condicionem ut senatus fauor apud principem aut prosit aut noceat?

À la différence de Durry, Mynors ne mentionne aucune conjecture et c'est sans sourciller qu'il met sous la plume du panégyriste la question suivante : «n'avonsnous pas assez souvent pu constater que la faveur du Sénat ou servait ou desservait celui sur lequel elle se posait ? ». Si besoin était, la suite montre que l'auteur a en vue le cas anormal où la faveur du sénat suscite la haine du prince. Pour obtenir le sens requis, on pourrait avec R. Novàk supprimer aut prosit aut, mais l'interpolation de ces mots est moins plausible que leur altération. Un peu plus loin (§ 4), Pline exprime la même idée d'une manière double : Non iam dissimulandus est amor ne noceat, non premendum odium ne prosit : eadem Caesar, quae senatus, probat improbatque. Il en allait peut-être de même au $\S 3$ : ut senatus fauor apud principem nOCeat, ODium prosit. La disparition de odium aurait entraîné le remembrement du passage qu'elle privait de sens, l'interversion des deux verbes afin de rapprocher prosit de fauor et l'insertion de la double disjonction aut... aut pour indiquer l'alternative. Mais une solution moins compliquée, c'est-à-dire impliquant un processus de faute plus simple, serait ita prosit ut noceat, « ne sert que pour nuire au bénéficiaire », cas de ita ut limitatif non reconnu et donc corrigé.

VI Panégyrique 90,6.

Habuerat hunc honorem periculis nostris diuus Nerua, ut nos, etsi minus ut bonos, promouere uellet, quia mutati saeculi signum et hoc esset quod florerent, quorum praecipuum uotum ante fuerat ut memoriae principis elaberentur. 
Littéralement « le divin Nerva avait payé aux dangers courus par nous (Pline) ces honoraires, à savoir qu'il voulait nous promouvoir, quoique moins comme personne méritante, parce qu'était un signe du changement des temps ce fait aussi que s'épanouissaient des gens dont le premier souhait avait auparavant été d'échapper au souvenir du prince ». Le rédacteur de l'article du TLL V,2 977,63 (publié en 1936), le grammairien J. B. Hofmann, admet une lacune - après etsi minus, je suppose. Sans connaître l'article du TLL, Durry 1938 critique l'hypothèse d'une lacune et le supplément de Juste Lipse etsi minus $<$ notos $>$, justifie etsi minus en comparant si minus... tamen et cite un passage très clair et très simple de Cicéron où tamen manque. Notre passage semble être le seul où etsi minus serait mis pour si minus « sinon... < du moins $>$ » et je suppose que c'est pour cette raison, en partie au moins, que Hofmann admet une lacune, que le sens n'impose pas. Durry traduit « non pour nos mérites, mais parce que... ». Etsi minus implique l'absence de prise en compte par Nerva des mérites justifiant la promotion du dignitaire. Il est plus plausible que minus exprime une idée relative et signifie que c'est moins pour ses mérites (par ailleurs reconnus et pris en compte) que comme signe du changement des temps que le prince voulait promouvoir Pline. Lire ut nos minus ut bonos promouere uellet quam quia... La perte de quam devant quia aura entraîné l'addition de etsi, dont l'usage comme incise devient de plus en plus fréquent dans la latinité postérieure ; j'imagine que c'est ainsi que l'entendait l'interpolateur présumé, « Nerva voulait nous promouvoir - quoique moins / non pour nos mérites - parce qu'il voyait un signe du changement des temps dans la promotion de ceux qui dans le passé désirait surtout vivre cachés ». Un auditeur a suggéré de garder etsi tout en ajoutant quam, mais nous croyons que le maintien de etsi avec quam est gauche et que cette gaucherie tranche avec l'élégance du passage - que dis-je ?, l'élégance constante, souvent raffinée et parfois maniérée, de tout le Panégyrique.

La prose souffre de beaucoup plus de chutes de mots que ne le supposent bien des éditeurs, plus prompts à supposer des apparitions de gloses que des disparitions de mots authentiques. Parmi les illustrations soumises aux auditeurs, certaines étaient empruntées à Quinte Curce et à un article publié dans Gnomon, 83, 2011, p. 499$507^{1}$; d'autres provenaient de la lecture de cet important document déjà sollicité, texte très beau, méconnu, à la tradition pauvre et médiocrement transmis ${ }^{2}$, le Panégyrique de Trajan. On a évoqué les cas suivants (lorsque la restitution est très simple, on se contente de l'indiquer par des crochets obliques sans la commenter) :

VII Panégyrique, 8,6.

Simul filius, simul Caesar, mox imperator et consors tribuniciae potestatis et omnia pariter et statim factus es, quae proxime parens uerus tantum in alterum filium contulit.

1. Il est suggéré p. 504 de cet article qu'il faut lire chez Quinte Curce, 7,3,10, non uites et arbores, si quae in tanto terrae rigore durare potuerunt, obruunt penitus. Hieme defossa $<e>$ latent, mais uites et arbores, si quae in tanto terrae rigore crescere potuerunt, penitus hieme defossae latent, où obruunt est supprimé par C. F. Kinch (1883). L'un des auditeurs, M. Barrière, remarque à juste titre qu'il y a un jeu sur les mots rigore et durare ( « résister »), lequel doit être conservé.

2. Nous n'avons pas vu l'édition critique de Rosario Moreno Soldevilla, Salamanque, 2010. 
Le passage paraît souffrir de plusieurs erreurs, que sa construction symétrique rend sensibles et dont elle fournit la solution :

Simul filius, simul Caesar, mox imperator et consors tribuniciae potestatis $<$ factus $\rangle$, omnia pariter et statim nactus es, quae proxime parens uerus sensim ${ }^{1}$ in alterum filium contulit.

L'absence chez Pline de nanciscor, suffisamment bien attesté en prose, ne paraît pas être une objection. La symétrie nactus / contulit semble heureuse.

VIII Panégyrique, 20,4.

Quam dissimilis nuper alterius principis transitus! si tamen transitus ille, non populatio fuit, cum abactus hospitium exereret, omniaque dextera laeuaque perusta et attrita, ut si uis aliqua uel ipsi illi barbari quos fugiebat inciderent.

La crux est notoire et l'explication de M. Haupt (Opuscula, III, p. 496), selon Durry 1938 "l'expulsion (des troupeaux) ouvrait le gîte », ne satisfait pas même Durry, qui cependant ne met pas le passage entre croix. Durry discute diverses propositions et trouve légitime l'hypothèse d'une lacune. Cette hypothèse paraît inévitable. Toute certitude est interdite et l'on en est réduit à des suggestions exempli gratia; en voici deux : cum abactus <hospes> hospitium exoraret, " du moment que l'hôte chassé quémandait l'hospitalité », cum abactus <hospes> hospitium <quaerens> exerraret (composé rare qui apparaît pour la première fois chez Stace, TLL V,2 1400,21-63). C'est avec joie qu'on constate avoir partiellement retrouvé une idée très plausible de W. S. Watt, The Classical Journal, 80, 1985, p. 340-341, cum abactus <hospes $>$ hospitium desereret, " the lodging-keeper was driven away and abandoned his lodginghouse ». Mais desereret exprime une idée déjà contenue dans abactus et dont la reprise n'offre qu'une platitude. La clausule offerte par exoraret / exerraret (dispondée) est reconnue par Baehrens fils (W. A. Baehrens, Panegyricorum Latinorum editionis novae praefatio maior. Accedit Plinii Panegyricus exemplar editionis, Groningue, 1910, p. 36-44), non l'hémiépès masculin formé par hospitium exereret ni le double choriambe formé par hospitium desereret, auquel Watt compare 12,2, pac|iscimur ut uicerimus. Toutefois, comme on sait, uicerimus peut former un ditrochée, clausule enregistrée par Baehrens fils. — « Deinde inciderint scribendum esse coniecit Schwarzius : rectius erit incidissent » (Haupt).

IX Panégyrique, 20,6.

Adsuescat imperator cum imperio calculum ponere; sic exeat, sic redeat tamquam rationem redditurus; edicat quid absumpserit. Ita fiet ut non absumat quod pudeat $<e>$ dicere. 6 Praeterea futuri principes, uelint nolint, sciant tanti tuum constat (...).

Crux notoire. Quanti <iter> suum constet pourrait convenir. Iter a naturellement été trouvé il y a longtemps (Rittershusius).

$\mathrm{X}$ Panégyrique, 24,2.

Manet imperatori quae prior oris humanitas. Incedebas pedibus, $<$ pedibus $>$ incedis ; laetabaris labore, <labore> laetaris.

1. Je n'exclus ni paulatim ni gradatim. Les trois adverbes sont chez Pline. 
On a peine à croire que Durry et Mynors n'aient pas corrigé deux erreurs aussi manifestes. On espère que les corrections qu'on offfe n'ont jamais été faites ou que, si elles l'ont été, elles n'ont pas été connues des deux éditeurs, car de les avoir négligées leur ôterait tout droit à l'indulgence. Mynors admet en 43,4 la correction évidente d'Ernesti Donauit pater tuus multa, <multa> et ipse donasti, qu'ignore Durry.

XI Panégyrique, 30,4.

Neque enim solum uagus ille (sc. Nilus) $<$ cum $>$ expandatur amnis intra usurpata semper collium substiterat atque haeserat, sed supino etiam ac detinenti solo non placido se mollique lapsu refugum abstulerat necdum satis umentes terras addiderat arentibus.

Tel est le texte de Durry et Mynors, où l'ajout de cum est une correction d'humaniste. Selon Durry 1938, cum expendatur causal explique uagus, mais il est clair que la notation est purement temporelle, le Nil vagabondant au moment de sa crue, et qu'il faut emprunter à l'humaniste qui ajoute cum son autre correction expanditur, non mentionnée par Mynors, qui pourtant dit de son auteur, $\mathrm{p}$. $\mathrm{x}$, cuius nomen pudet me nescire; (...) longe plura admirabili sollertia emendauit. « Non seulement ce fleuve, traduit - avec aussi peu d'exactitude que d'élégance - Durry, qu'on peut dire errant puisqu'il déborde, s'était arrêté et fixé en deçà du niveau où il envahissait toujours les collines, mais aussi du terrain en pente douce et qui devrait le retenir, ce n'est pas d'un glissement paisible et lent, mais en fuyard qu'il s'était retiré, et les terres insuffisamment rafraîchies s'étaient ajoutées par sa faute aux tout-à-fait arides ». L'usage demande non refugum, mais refugus et Durry et Mynors auraient dû adopter cette correction de Baehrens père (Teubner, Leipzig, 1874), que Mynors ne cite même pas. Durry 1938 se bat les flancs pour expliquer detinenti : «detinenti est expliqué par supino; des colles l'eau se retire toujours vite; les parties basses devraient la retenir ». Mais detinenti, si conatif que le participe puisse être, ne peut pas signifier « qui devrait le retenir ». C'est plutôt devant detinenti que devrait se trouver la négation qui affecte placido mollique lapsu : refugus n'implique nullement une décrue violente; il est au contraire plausible qu'à la crue paresseuse évoquée au $\S 2$ (piger Nilus cunctanter alueo sese ac languide extulerat) correspond une décrue du même type. Baehrens père n'a donc pas démérité en transférant non, même si une autre solution est possible et même préférable : perte d'un adverbe (alioqui, « en général »; semper ?) devant detinenti et, indépendamment, ajout de non à cause d'une conclusion erronément tirée de refugum. Baehrens, je le soupçonne, pensait que non detinenti cadrait bien avec supino pris au sens de « légèrement incliné », mais supino solo désigne ici l'étendue plane, en opposition aux colles, et l'idée est non que la déclivité du sol explique la non-rétention de l'eau mais que, contrairement à l'habitude, la plaine ne s'imbibe pas parce que le fleuve s'en retire. Ensuite, usurpata collium est, Durry le note, tout à fait inusuel, et usurpata semper collium pour semper usurpatos colles l'est encore davantage : c'est même, observe Madvig (Adversaria critica, III, p. 217-218), un solécisme. La perte de $s$ final devant semper et le passage de $-O$ à $-a$ ont peut-être amené le génitif collium. Enfin, le latin exprime précisément au moyen de citra la notion que le texte transmis et les traducteurs assignent à intra. On peut certes citer quelques passages (cf. TLL VII,2 $38,73-39,9)$ où l'on trouve intra dans le sens de citra, mais leur autorité n'est pas 
incontestable, soit que ces passages ne soient pas comparables soit que la même faute y soit envisageable. On suggère donc, au moins à titre d'exemple, le texte suivant :

Neque enim solum uagus ille $<$ cum $>$ expanditur amnis citra superfuso $<s>$ semper colles substiterat atque haeserat, sed supino etiam atque $<$ alioqui $>$ detinenti solo [non] placido se mollique lapsu refugus abstulerat necdum satis umentes terras addiderat arentibus.

« Non seulement ce fleuve connu pour vagabonder en période de crue s'était arrêté et immobilisé en deçà des collines qu'il inondait toujours, mais, de surcroît, il s'était, dans le reflux d'un cours paisible et mesuré, retiré du sol plan qui retenait généralement l'eau, et il avait ajouté des terres encore insuffisamment irriguées à celles restées arides ». Pour superfusus dit non du liquide répandu mais de ce sur quoi il est répandu, voir l'OLD s. v. superfundo $1 \mathrm{~b}$ et comparer, pour cette liberté de construction, d'autres composés de fundo. La faute usurpat- $>$ superfus- proviendrait de la métathèse de plusieurs lettres.

XII Panégyrique, 52,6.

Simili reuerentia, Caesar, non apud genium tuum bonitati tuae gratias agi, sed apud numen Iouis Optimi Maximi pateris : illi debere nos <uis> quidquid debeamus, illius, quod bene facias, muneris esse qui te dedit.

Sans l'addition de uis, il manque un verbe dont debere et esse puissent dépendre d'une manière plausible (car le sens de pateris le rend impropre à régir les propositions infinitives juxtaposées dont le mot initial est une forme du démonstratif ille).

XIII Panégyrique, 55,4.

Age, siquando pietas nostra silentium rupit et uerecundiam tuam uicit, quae qualiaque aut decernimus nos, aut tu non recusas! - ut adpareat non superbia et fastidio te amplissimos honores repudiare, qui minores non dedigneris.

«S'il arrive que notre reconnaissance rompe le silence et triomphe de votre modestie, rappelons-nous quels honneurs nous vous décernons, et quels honneurs vous ne refusez pas : on verra que ce n'est point par orgueil et par dédain que vous rejetez les plus grands, puisque vous ne dédaignez pas les moindres » (J.-L. Burnouf, auteur d'une élégante traduction annotée du Panégyrique, Paris, 1824). La ponctuation de l'édition Mynors facilite l'intelligence du texte, mais l'exclamative et la consécutive suivante forment un enchaînement très imparfait et douteux : des mots semblent manquer entre recusas et ut adpareat, peut-être SATIS SVNT, « la nature des honneurs que nous te décernons et de ceux que tu ne refuses pas suffit à montrer que ce n'est pas par orgueil et par dédain que tu refuses les plus grands $»$.

XIV Panégyrique, 67,6.

Alii se superstites rei publicae optabant faciebantque $<$ ut essent $>$, tibi salus tua inuisa est, si non sit cum rei publicae salute coniuncta.

XV Panégyrique, 84,5.

Neque enim umquam periclitabuntur <rursus $>$ esse priuatae quae non desierunt.

Le panégyriste parle de Plotina et de Marciana. 
Le texte du Panégyrique souffre aussi naturellement d'autres types de fautes, dont voici, parmi ceux qu'on a étudiés, quelques exemples :

XVI Panégyrique 13,4.

Tunc enim illum imitationis ardor semperque melior aliquis accenderet.

Au temps des Fabricius, des Scipions et des Camilles, Pline jugerait moins admirable la belle conduite d'un empereur, car alors il y avait matière à imitation. Imitation? plutôt émulation, aemulationis (cf. Lettres, 8,6,13).

XVII Panégyrique, 22,2.

Tu sola corporis proceritate elatior aliis et excelsior non de patientia nostra quendam triumphum sed de superbia principum egisti.

Altior paraît évident ( $<$ latior $<$ elatior avec dittographie de la finale du mot précédent).

XVIII Panégyrique, 30,2.

Haec (sc. Aegyptus) inopina siccitate usque $<$ in $>$ iniuriam sterilitatis exaruit, quia piger Nilus cunctanter alueo sese ac languide extulerat, ingentibus quidem tunc quoque ille fuminibus, fluminibus tamen conferendus.

Lire ingentibus ille quidem tunc quoque fluminibus conferendus. C'est Heinrich Keil qui a remis ille à sa vraie place; nous avons suggéré de supprimer fluminibus tamen comme explication insérée avant d'apprendre de Baehrens fils que cette correction avait déjà été faite par G. Suster. Baehrens fils et, à sa suite, Durry ont tout à fait tort de défendre le texte transmis en invoquant 24,4, Emines, excellis ut honor, ut potestas, quae super homines quidem, hominum sunt tamen, ou d'autres passages du même type. Durry traduit « et si même alors il était encore comparable aux plus grands fleuves, il ne leur était plus incomparable ». Pour justifier fluminibus tamen conferendus, il est obligé d'inventer pour cette séquence un sens qui justifie le correctif introduit par tamen, mais on se demande bien comment conferendus, pris avec la première occurrence de fluminibus, signifie «comparable» et, pris avec la seconde, «ne... plus incomparable». C'est sur sa traduction abusive que Durry fonde son explication de fluminibus tamen conferendus et c'est d'après cette explication qu'il justifie le texte. On ne saurait guère trouver d'exemple plus instructif de dévoiement de la fonction de la traduction.

XIX Panégyrique, 61,7.

Vterque nuper consulatum alterum gesserat a patre tuo (id est quanto minus quam a te) datum.

Selon Durry 1938, quanto minus est mis pour paulo minus, dont il croit bon d'illustrer l'usage. Mais quanto minus dit plutôt le contraire de paulo minus et quanto s'est ici substitué à la vraie leçon paulo.

XX Panégyrique, 75,6.

Quid nunc ego super ea quae sum cum toto senatu precatus pro senatu precer, nisi ut haereat animo gaudium quod tunc oculis protulisti, ames illum diem et tamen uincas, noua merearis, noua audias? eadem enim dici nisi facta... non possunt. 
Tel est le texte de l'importante édition de Heinrich Keil (Teubner, 1870), célèbre pour l'index prosopographique que Théodore Mommsen a confectionné pour elle. Keil mentionne la correction souvent adoptée nisi ob eadem facta de Cataneus et la sienne, nisi facta sint eadem, qui n'améliore pas la précédente. R. Moreno Soldevilla (The Classical Quarterly, 60, 2010, p. 287) défend le texte transmis, comprend « for the same things cannot be said unless they are done » et compare 2,8, alternisque uotis " haec faciat, haec audiat " quasi non dicturi nisi fecerit comprecamur! Mais Pline veut certainement dire que de nouveaux compliments impliquent de nouveaux actes et ni le texte transmis ni les corrections de Cataneus et de Keil ne sauraient exprimer cette idée. Il faudrait à la rigueur noua enim dici nisi facta (sc. sunt) non possunt ou noua enim dici nisi noua facta (sc. sunt) non possunt. La disparition de la troisième occurrence de noua aura amené le faux bouche-trou eadem.

\section{Panégyrique, 83,1.}

Habet hoc primum magna fortuna quod nihil tectum, nihil occultum esse patitur.

«La grande fortune a cela de particulier...» (Durry). C'est exactement cela : il faut donc proprium (abréviation mal comprise). Cette correction qui aurait dû s'imposer date de Rittershusius (1560-1613)!

\section{Panégyrique, 95,2.}

Vos proxime destinationem consulatus mei his acclamationibus adprobauistis ut intellegam etiam atque etiam enitendum mihi ut hunc consensum uestrum complectar et teneam et in dies augeam.

Pline sénateur s'adresse à ses pairs. Burnouf traduit complectar « justifier votre estime », Durry 1947 «mériter votre approbation », idée qu'exprimerait commerear. Le mot n'est pas ailleurs chez Pline et le déponent est rare mais suffisamment attesté pour être au moins possible chez Pline dans le sens de «mériter » (TLL III 1880,51-57). Dans son commentaire de 1938, Durry renvoyait à la rubrique du $T L L$ III 2085,45-53, qui, sans mentionner notre passage, en cite d'autres où complector vaut comprehendere, capere, arripere, occupare, sibi uindicare, potiri aliqua re. Pline dirait donc comprendre qu'il doit s'efforcer sans relâche d'obtenir, de conserver, de renforcer le consensus de ses pairs. Cela se tient. On termine donc ce voyage critique chez Pline avec un passage qui illustre le fait qu'une meilleure intelligence du texte transmis, naturelle, non forcée et conforme à l'usage, peut rendre inutile le recours à la conjecture. 